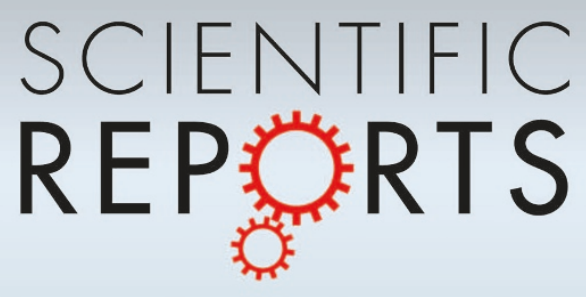

SUBJECT AREAS:

GENETIC ASSOCIATION STUDY

\title{
Association of genetic variants of GRIN2B with autism
}

MOLECULAR BIOLOGY

Yongcheng Pan ${ }^{1 *}$, Jingjing Chen ${ }^{1}$, Hui Guo ${ }^{1,2}$, Jianjun Ou' ${ }^{2}$, Yu Peng', Qiong Liu', Yidong Shen², Lijuan Shi' ${ }^{2}$, Yalan Liu' ${ }^{1,3}$, Zhimin Xiong ${ }^{1,4}$, Tengfei Zhu' ', Sanchuan Luo' , Zhengmao Hu' , Jingping Zhao ${ }^{2}$ \& Kun $\mathrm{Xia}^{1,5,6}$

Received

23 September 2014

Accepted

14 January 2015

Published

6 February 2015

Correspondence and requests for materials should be addressed to

H.G. Iguohui@sklmg. edu.cn) or K.X. (xiakun@sklmg.edu.

$\mathrm{cn})$

* These authors contributed equally to this work. To evaluate genetic variants of GRIN2B in autism etiology, we performed a system association study of sample of 1,945 subjects. Meta-analysis of a triad family cohort and a case-control cohort identified Sanger sequencing of 275 probands from a triad cohort identified several variants in coding regions, that common variants and related haplotypes of GRIN2B are associated with autism risk.

\author{
${ }^{1}$ The State Key Laboratory of Medical Genetics, School of Life Sciences, Central South University, Changsha, Hunan, China, ${ }^{2}$ Mental \\ Health Institute, The Second Xiangya Hospital, Central South University, Hunan, China, ${ }^{3}$ The Xiangya Hospital, Central South \\ University, Hunan, China, ${ }^{4}$ The Third Xiangya Hospital, Central South University, Hunan, China, ${ }^{5}$ College of Life Science and \\ Technology, Xinjiang University, Xinjiang, China, ${ }^{6}$ Key Laboratory of Medical Information Research (Central South University), \\ Changsha, Hunan, China.
}

Autism (MIM 209850) is a complex neurodevelopmental disorder characterized by social communication impairments and restricted repetitive behaviors. It has a high heritability, although much remains unclear. common and rare variants of GRIN2B and autism in cohorts from a Chinese population, involving a total significant associations of multiple common variants and autism risk $\left(\mathrm{P}_{\min }=1.73 \times 10^{-4}\right)$. Significantly, the haplotype involved with the top common variants also showed significant association $\left(P=1.78 \times 10^{-6}\right)$. including four common variants and seven rare variants. Two of the common coding variants were located in the autism-related linkage disequilibrium (LD) block, and both were significantly associated with autism $\left(\mathrm{P}<9 \times 10^{-3}\right)$ using an independent control cohort. Burden analysis and case-only analysis of rare coding variants identified by Sanger sequencing did not find this association. Our study for the first time reveals

A utism (OMIM\#209850) is a complex neurodevelopmental disorder, characterized by social and language communication impairments and restricted repetitive patterns of behavior ${ }^{1}$. It appears in early childhood, with a typical onset before the age of 3 years old, and shows a remarkable sex bias, with a male excess estimated at 3-4:12,3. The prevalence of autism spectrum disorders has risen to 1 in 68 according to the most recent estimates reported by the United States Centers for Disease Control and Prevention ${ }^{4}$. While it is believed that both genetic and environmental factors contribute to the etiology of autism, a recent study revealed that the narrow-sense heritability of autism is approximately $52.4 \%$, which is mostly attributed to common genetic variants or their interactions with environmental factors ${ }^{5}$. Rare de novo mutations contribute substantially to individual liability, but their contribution to variance in liability is only $2.6 \%{ }^{5}$.

De novo loss-of-function mutations have been recurrently identified by exome sequencing at several genes, including GRIN2B. Tarabeux et al. first identified one de novo mutation of GRIN2B in a patient with autism ${ }^{6}$. Subsequently, O'Roak et al. identified three de novo loss-of-function mutations and one de novo missense mutation of GRIN2B using exome and targeted sequencing ${ }^{7}$. The observed number of de novo mutation events was significantly higher at GRIN2B than expected on the basic of the mutation rates estimated for each gene ${ }^{8}$.

GRIN2B encodes an NR2 subunit of N-methyl-d-aspartate receptors (NMDARs), a major class of excitatory glutamate receptors in the central nervous system. NMDARs are thought to be tetramers, assembling as a pair of dimers formed from NR1, NR2 and NR3 subunits. The NR2 subunit (GRIN2A, GRIN2B, GRIN2C, or GRIN2D) is the predominant excitatory neurotransmitter receptor in the mammalian brain, acting as the agonist -binding site for glutamate ${ }^{9}$. Disruption of NMDARs causes abnormal synaptogenesis and an imbalance between excitatory and inhibitory currents, which is important for the pathogenesis of autism ${ }^{10,11}$. While de novo rare mutations of GRIN2B have been identified in autism patients, common variants and rare inherited variants have not yet been systematically investigated. In this study, we examined the association of common and rare variants of GRIN2B with autism risk in Han Chinese populations. 
We performed an association analysis in two sample cohorts to search for common variants associated with autism. One cohort, consisting of 275 case-parents triad families $(n=825)$, was analyzed using a transmission disequilibrium test (TDT); the other cohort, consisting of cases and controls $(n=1,120)$, was analyzed using logistic regression (method). A meta-analysis of the two cohorts was performed using the Stouffer combined method to obtain combined evidence for genetic associations with autism. Sanger sequencing was then conducted on 275 probands from the triad families (methods). Common variant association analysis of the coding variants was performed using an independent control cohort. Burden and case-only analyses were evaluated for the rare variants identified by Sanger sequencing.

\section{Results}

Common variants and related haplotypes are associated with autism. In total, 74 single-nucleotide polymorphisms (SNPs) were included for the association analysis after strictly quality controls (method) in both case-parents triad family and case-control cohort. All SNPs were located in non-coding regions. TDT analysis

Table 1 | Results of single point association analysis for the triad family cohort and the case-control cohort and combined meta-analysis

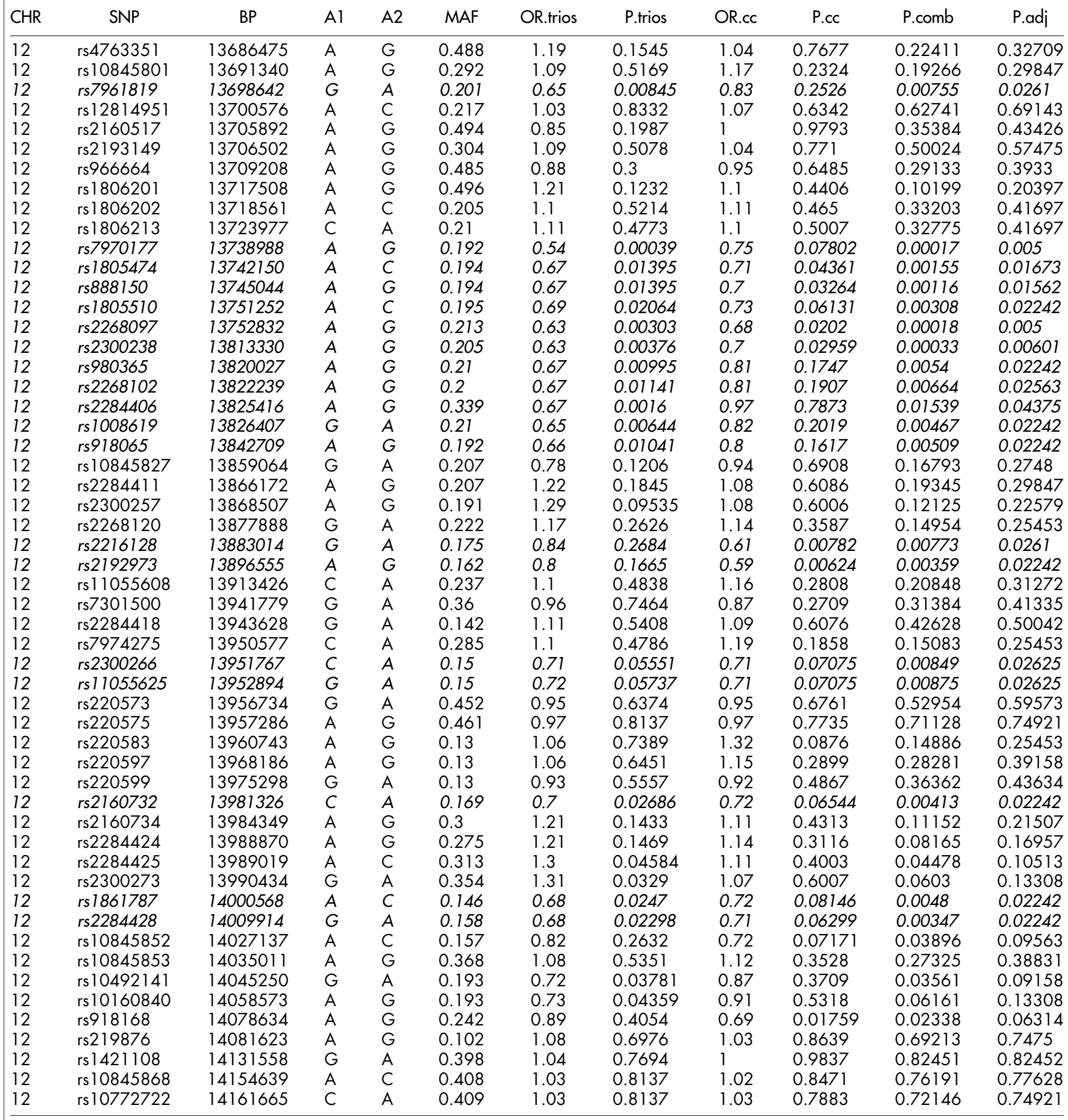



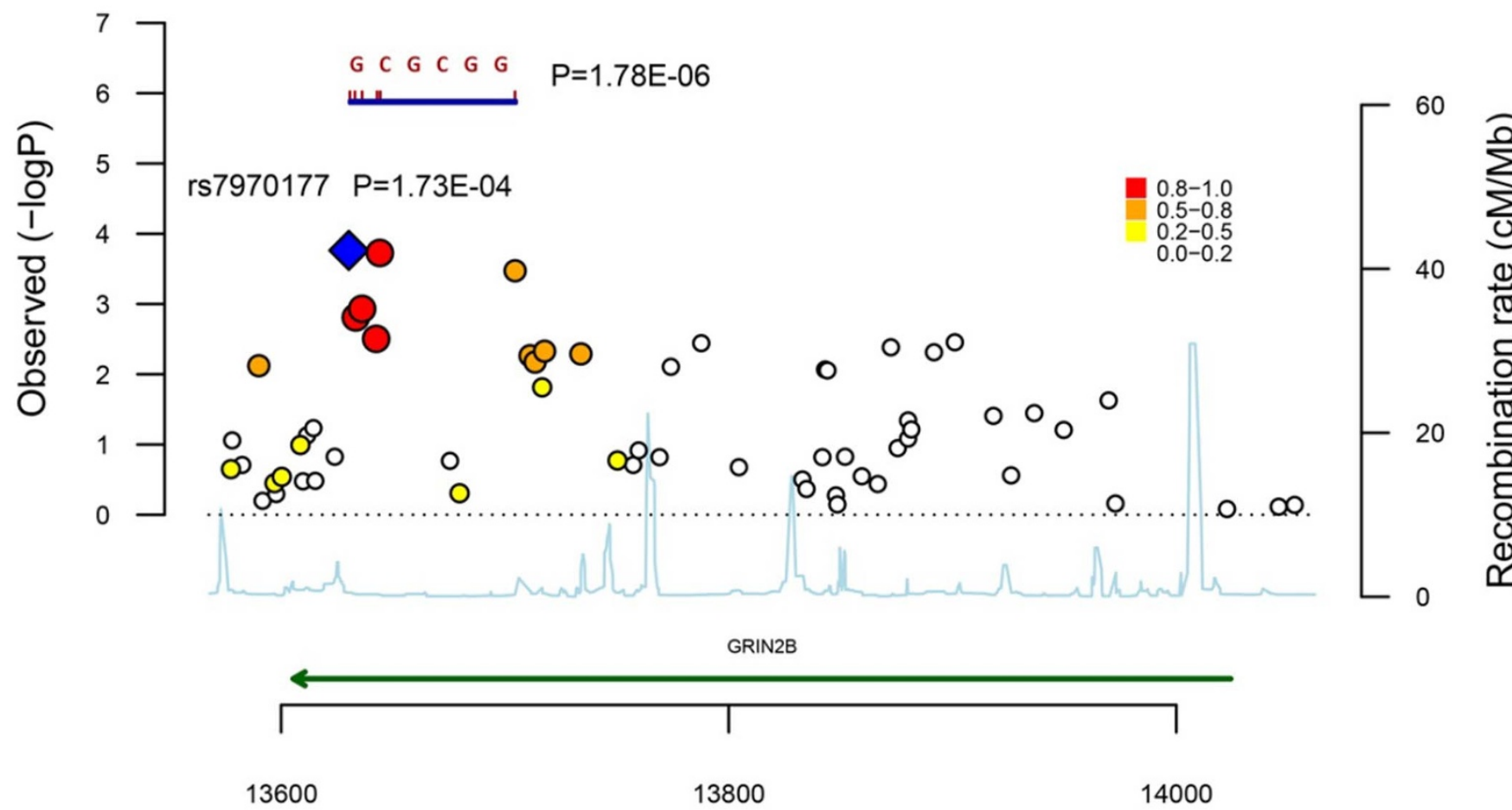

\section{Chromosome 12 position (kb)}

Figure 1 Regional association plot of a negative logarithm of combined P-values for GRIN2B common variants. The most significant SNP was rs7970177 ( $\mathrm{P}=1.73 \mathrm{E}-04)$, which showed strong LD with its nearby five SNPs $\left(\mathrm{r}^{2}>0.8\right)$. The six SNPs constructed a strong LD block and showed strong associations with autism $(\mathrm{P}=1.78 \mathrm{E}-06)$.

of the triad family cohort identified 19 SNPs with nominal significance associations (P.trios $<0.05$, Table 1). Logistic regression analysis of the case-control cohort identified seven SNPs showing significant associations (P.cc $<0.05$, Table 1 ). To validate the association results and to reduce the possible false positives, we combined the results of the two cohorts for metaanalysis, and 23 SNPs showed significant associations (P.comb < 0.05 , Table 1, Figure 1). Of these, 19 SNPs showed significant associations after correcting for multiple testing (P.adj $<0.05$,
Table 1$)$. Most of the significantly associated SNPs $(n=11)$ were located in a LD block (Table 1, Figure 2). Therefore, we performed haplotype association analysis using the sliding-widow method in PLINK, followed by meta-analysis. The most significant haplotype, GCGCGG, was observed at six SNPs in strong LD (rs7970177| rs1805474|rs888150|rs1805510|rs2268097|rs2300238, $\mathrm{D}^{\prime}>0.9$, $\mathrm{r}^{2}>0.8, \mathrm{P}=1.78 \mathrm{E}-06$ ). (Table 2, Figure 1). In addition to the SNPs located in the LD block, there were also five independent association signals (Table 1), including rs7961819 $(\mathrm{P}=0.0261)$,
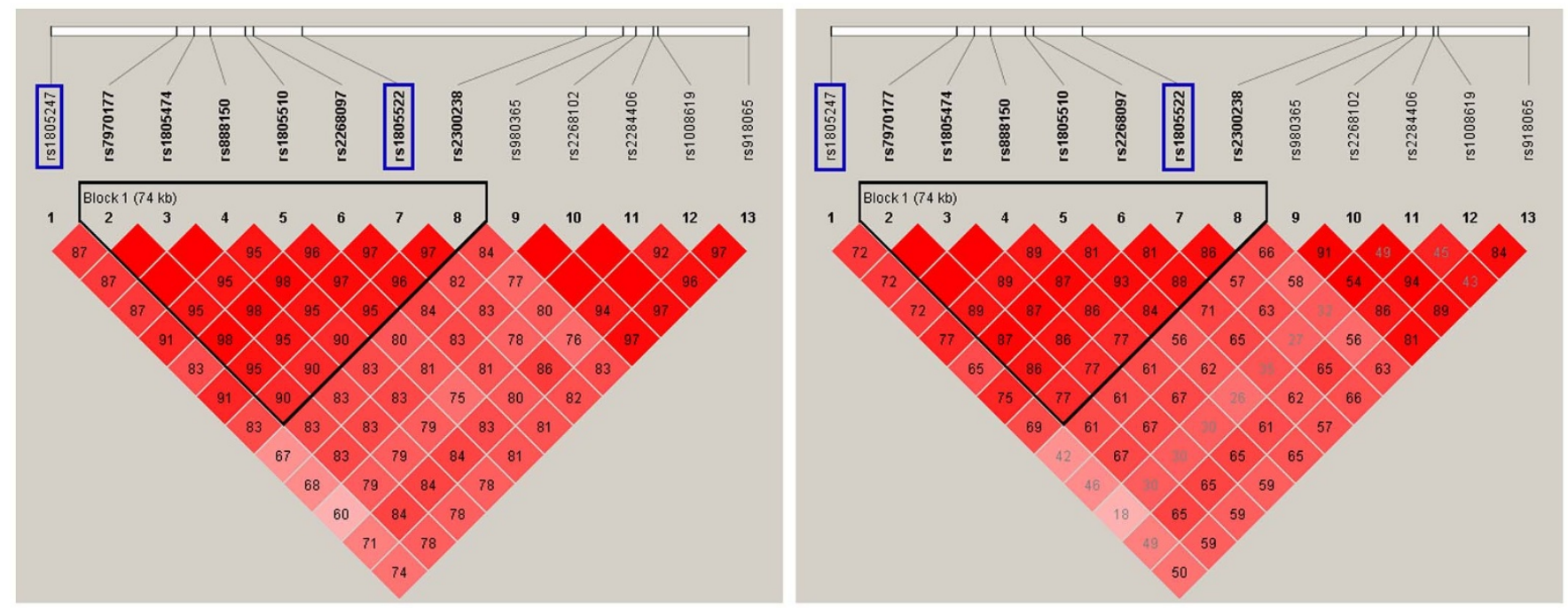

Figure $2 \mid$ Haplotype plot for the LD block constructed from 11 significant SNPs. SNPs with blue squares were identified by Sanger sequencing and showed significant association. The six SNPs included by the black triangle (Block 1) constructed the most significant haplotype identified by slidingwindow analysis. 


\begin{tabular}{|c|c|c|c|c|c|c|c|c|c|c|c|}
\hline HAPLOTYPE & FREQ.tios & FREQ.cc & F_A.cc & F_U.cc & T.trios & U.trios & CHISQ.cc & CHISQ.trios & P.cc & P.trios & P.comb \\
\hline $\bar{G}$ & 0.824 & 0.813 & 0.8494 & 0.8077 & 91 & 49 & 3.106 & 12.60 & 0.07802 & $3.86 \mathrm{E}-04$ & $1.73 \mathrm{E}-04$ \\
\hline GCG & 0.806 & 0.810 & 0.8576 & 0.8074 & 107.1 & 58.93 & 4.496 & 13.98 & 0.03397 & $1.84 \mathrm{E}-04$ & $3.42 \mathrm{E}-05$ \\
\hline GCGC & 0.800 & 0.800 & 0.8537 & 0.8038 & 109.1 & 59.93 & 4.365 & 14.31 & 0.03667 & $1.55 \mathrm{E}-04$ & $3.29 \mathrm{E}-05$ \\
\hline GCGCG & 0.776 & 0.787 & 0.856 & 0.7978 & 119.1 & 63.93 & 5.773 & 16.64 & 0.01628 & $4.52 \mathrm{E}-05$ & $4.58 \mathrm{E}-06$ \\
\hline GCGCGGGG & 0.758 & 0.761 & 0.8372 & 0.7904 & 126.1 & 65.95 & 3.546 & 18.83 & 0.05968 & $1.43 \mathrm{E}-05$ & $1.08 \mathrm{E}-05$ \\
\hline GCGCGGGGG & 0.637 & 0.627 & 0.6764 & 0.6541 & 167.1 & 98.39 & 0.573 & 17.77 & 0.44920 & 2.49E-05 & 4.38E-04 \\
\hline GCGCGGGGGA & 0.636 & 0.625 & 0.6719 & 0.6537 & 167.1 & 97.38 & 0.384 & 18.36 & 0.53530 & $1.82 \mathrm{E}-05$ & 5.23E-04 \\
\hline GCGCGGGGGAG & 0.636 & 0.625 & 0.672 & 0.6565 & 167.1 & 97.39 & 0.278 & 18.36 & 0.59810 & $1.83 \mathrm{E}-05$ & 6.67E-04 \\
\hline
\end{tabular}

Note: Haplotype GCGCGGGGGAG involved SNPs are

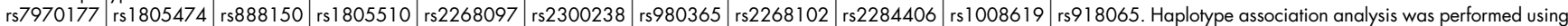
PLINK with a sliding window. P.cc represents P values of the case-control cohort; P.trios represents P values of the trios cohort; P.comb represents P values after meta-analysis.

rs2216128|rs2192973 $\left(\mathrm{P}=0.0261\right.$ and $\mathrm{P}=0.02242, \mathrm{r}^{2}=0.9$, $\left.\mathrm{D}^{\prime}=0.997\right), \mathrm{rs} 2300266 \mid \mathrm{rs} 11055625\left(\mathrm{P}=0.02625, \mathrm{r}^{2}=0.993\right.$, $\left.\mathrm{D}^{\prime}=0.997\right), \mathrm{rs} 2160732(\mathrm{P}=0.02242)$ and $\mathrm{rs} 1861787 \mid \mathrm{rs} 2284428$ $\left(\mathrm{P}=0.02242, \mathrm{r}^{2}=0.870, \mathrm{D}^{\prime}=0.979\right)$.

Sanger sequencing of the coding and splicing regions in the 275 triad probands identified four common (minor allele frequency $[\mathrm{MAF}]>0.05)$ coding variants, all of which were synonymous (Table 3). To determine whether these coding common variants are associated with autism, we performed association analysis by logistic regression using Asian samples (CHB, CHS and JPT) from the 1000 genome project as controls. Two variants showed significant associations (c.T4197C, rs1805247, MAF $=0.2028, \mathrm{P}=0.0015$, odds ratio $[\mathrm{OR}]=0.59 ; \mathrm{c} .1806 \mathrm{C}>\mathrm{T}, \mathrm{rs} 1805522, \mathrm{MAF}=0.1871$, $\mathrm{P}=0.0042$, $\mathrm{OR}=0.62$; Table 3 ). The association was still significant after correcting for multiple testing (c.T4197C, P.adj $=0.0061$; c. $1806 \mathrm{C}>\mathrm{T}$, P.adj $=0.0083$ ). These two variants were in strong $\mathrm{LD}\left(\mathrm{D}^{\prime}=0.91, \mathrm{r}^{2}=0.75\right)$ and were located in the autism-related LD block identified above (Figure 2). Both $\operatorname{rs} 1805247\left(\mathrm{D}^{\prime}=0.87, \mathrm{r}^{2}=\right.$ $0.72)$ and $\mathrm{rs} 1805522\left(\mathrm{D}^{\prime}=0.95, \mathrm{r}^{2}=0.86\right)$ were in strong $\mathrm{LD}$ with the top association signal (rs7970177) of the autism-related LD block. This result further validated the association of this haplotype with autism risk.

Rare variants of GRIN2B are not associated with autism risk. In addition to common variants identified in the coding regions by Sanger sequencing, we also identified seven rare coding variants (MAF $<0.01$ ), including four synonymous variants and three missense variants (Table 4). Two missense variants (c.A4015G:p.M1339V, c.C3818A:p.T1273K) were not reported (dbSNP138 and ESP6500). Both were inherited from an asymptomatic father. To test whether rare variants of GRIN2B are associated with autism risk, we first performed burden analysis using Asian samples (CHB, CHS and JPT) from the 1000 genome project as controls. Burden analysis identified no significant difference in the burden of rare variants between cases and controls $(P=0.42$, Table 4). We then performed a uniq (case-only) analysis to test whether autism patients carried more case-uniq variants. However, no significance was observed $(\mathrm{P}=0.47$, Table 4$)$.

\section{Discussion}

In this study, both TDT analysis of the triad family cohort and regression analysis of the case-control cohort identified multiple SNPs with significant associations. After further meta-analysis by combining the results from both cohorts and correcting for multiple testing, 19 SNPs showed significant associations. Importantly, 11 SNPs were located in a LD block. The six SNPs with the GCGCGG haplotype were strongly associated with autism.

Sanger sequencing of the coding and splicing regions in the 275 triad probands identified four common variants. Association analysis confirmed two significant associated variants, rs1805247 and rs1805522. Variant rs1805522 was located between the first and second transmembrane segment (M1 and M2, respectively). M1 and M2, combined with a pore helix and pore loop, form the narrowest part of the ion channel pore, which determines the narrow constriction and ion selectivity of the channel ${ }^{12}$. Variant rs 1805247 was located at a conserved carboxy-terminal domain (CTD), which has an important role in its interaction with specific signaling proteins, such as CaMKII, SAP102, PSD-95, $\alpha$-Actinin and Ras-GRF1 ${ }^{13}$. These two variants were located in the LD block constructed by 11 significant SNPs. These results further validated the association of the haplotype with autism risk. Interestingly, Yoo et al. reported a five-SNP haplotype association of GRIN2B with autism in Koreans ${ }^{12}$, and their associated haplotype shared the same SNPs rs1805247 and rs 1805522 with our results. All evidence indicated that multiple common variants of GRIN $2 B$ and related haplotypes were associated with autism risk.

Sanger sequencing also identified two missense variants (c.A4015G:p.M1339V, c.C3818A:p.T1273K) that were inherited from an asymptomatic father. These missense variants were also located in the conserved intracellular CTD. It was reported that GRIN2B C-terminally truncated mice die shortly after birth; the lethal phenotype of NR2B C-terminally truncated mice might be caused by impaired intracellular signaling due to the missing intracellular receptor domain ${ }^{14}$. Further investigation is still needed.

Dozens of genome-wide association studies have revealed that coding synonymous variants or common variants lying outside of

\begin{tabular}{|c|c|c|c|c|c|c|c|c|}
\hline c.C2664T:p.T888T & synonymous & 0.216 & 0.484 & 0.496 & rs1806201 & 1.05 & 0.6849 & 0.6849 \\
\hline c.C1806T:p.|602I & synonymous & 0.039 & 0.187 & 0.123 & rs 1805522 & 0.62 & 0.0042 & 0.0083 \\
\hline c.C4218T:p.F1406F & synonymous & 0.027 & 0.077 & 0.092 & rs1805246 & 1.23 & 0.3570 & 0.4760 \\
\hline
\end{tabular}

Note: MAF_1000G only included Chinese samples (CHB and CHD). P.adj represents adjusted P values using FDR. 


\begin{tabular}{|c|c|c|c|c|c|c|c|}
\hline AAChange & ExonicFunc & num_ESP6500 & num_1000G & num_275case & dbSNP138 & burden & case-only \\
\hline c.C2793T:p.V93 IV & synonymous & 0 & 0 & 1 & novel & $P=0.42$ & $P=0.47$ \\
\hline c.C2877T:p.F959F & synonymous & 0 & 0 & 3 & novel & & \\
\hline c.A3429G:p.S1143S & synonymous & 0 & 0 & 6 & novel & & \\
\hline c.C3564G:p.G1188G & synonymous & 0 & 0 & 3 & novel & & \\
\hline c.C3683T:p.T1228M & missense & 0 & 0 & 1 & rs75670883 & & \\
\hline c.A4015G:p.M1339V & missense & 0 & 0 & 1 & novel & & \\
\hline c.C3818A:p.T1273K & missense & 0 & 0 & 1 & novel & & \\
\hline
\end{tabular}

protein-coding regions are functional $1^{15}$. Although we cannot determine the specific biological significance of the significant variants we identified in the current study, they may be located in gene regulation elements; however, this possibility remains unconfirmed. For example, the variants might be involved in the risk of autism by regulating GRIN2B expression. Further study should be conducted to reveal the functional consequences of these variants as related to autism risk.

\section{Methods}

Subjects. Subjects used for the common variants association study included one cohort of 275 case-parent triad families and one cohort of case-controls $(n=1,120)$ from the Chinese population. The detailed sample recruitment and diagnosis was described in our previous paper ${ }^{16}$. In summary, all patients were diagnosed with the Diagnostic and Statistical Manual of Mental Disorders-IV criteria (DSM-IV-TR) for autistic disorder by senior psychiatrists from the Psychiatric department of the Second Xiangya Hospital. Patients with fragile $\mathrm{X}$ syndrome, tuberous sclerosis, chromosomal abnormality, dysmorphic features, or any other neurological conditions suspected to be associated with autism were excluded. In addition, none of the patients was known (according to the parents' reports) to have any other abnormalities. Subjects used for Sanger resequencing for the coding regions included 275 patients from the 275 triad families in the above common variants association study. All participants provided written informed consent. This study was approved by the institutional review board at the State Key Laboratory of Medical Genetics. All methods were performed in accordance with approved guidelines.

Genotyping, quality control and Sanger resequencing. All autism cases and controls were genotyped using the Illumina HumanHap CNV370Quad BeadChip or Illumina HumanHap 610Quad BeadChip, as described in our previous paper. Detailed genotyping, quality controls and population stratification analysis were also described. We selected variants within in a 30-kb distance of GRIN2B gene regions. There are 100 variants in the Illumina HumanCNV370Quad BeadChip within this region. After a series of quality controls (SNPs were zeroed out if Mendelian errors $>5 \%$, genotype rate $>5 \%$ and minor allele frequency $>0.05$ ), 74 variants remained for association analysis.

For the 275 probands for Sanger resequencing, all exons, flanking splicing sites and untranslated regions (UTRs) of the GRIN2B gene (NM_000834.3) were amplified by polymerase chain reaction (PCR). PCR primers were designed using the online Primer3 program (http://frodo.wi.mit.edu/). The PCR products were verified by $6 \%$ polyacrylamide gel electrophoresis. Sanger sequencing was performed using an ABI 3100/3130 DNA analyzer. All identified variants were confirmed by repetitive independent PCR amplification and DNA bidirectional sequencing.

Statistical analysis. Common variant association analysis was performed using PLINK $^{17}$. The TDT was used for the case-parent triad cohort, and logistic regression analyses were used for the case-control cohort. The combined $\mathrm{P}$ values from both cohorts were calculated using Stouffer's Z-score method for meta-analysis. The haplotype analysis was performed using up to 10-SNP sliding window approach, followed by meta-analysis of haplotype association results.

Case-control association analyses for the common coding variants identified by Sanger sequencing were performed using logistic regression analysis in PLINK. Rare variants identified by Sanger sequencing were analyzed using PLINK/SEQ (http:// atgu.mgh.harvard.edu/plinkseq/index.shtml). Chinese samples from the 1000 genome project ( $\mathrm{CHB} \& \mathrm{CHD}, \mathrm{n}=286$ ) were selected as controls in the above analysis. The false-discovery rate (FDR) procedure, proposed by Benjamini and Hochberg (1995), was applied for handling multiple comparisons problems.

The regional association plot and haplotype plots were generated using R (http:// www.r-project.org/) and Haploview ${ }^{18}$, respectively.

1. American Psychiatric Association. Diagnostic and Statistical Manual of Mental Disorders. Fifth edn, Arlington,VA: American Psychiatric Publishing. (2013).
2. Levy, S. E., Mandell, D. S. \& Schultz, R. T. Autism. Lancet. 374, 1627-1638 (2009)

3. Manning-Courtney, P. et al. Autism spectrum disorders. Curr Probl Pediatr Adolesc Health Care. 43, 2-11 (2013).

4. Autism and Developmental Disabilities Monitoring Network Surveillance Year Principal Investigators; Centers for Disease Control and Prevention. Prevalence of autism spectrum disorders--Autism and Developmental Disabilities Monitoring Network, 14 sites, United States, 2008. MMWR Surveill Summ. 61, 1-19 (2012).

5. Gaugler, T. et al. Most genetic risk for autism resides with common variation. Nat Genet. 46, 881-885 (2014).

6. Tarabeux, J. et al. Rare mutations in N-methyl-D-aspartate glutamate receptors in autism spectrum disorders and schizophrenia. Transl Psychiatry. 1, e55 (2011).

7. O'Roak, B. J. et al. Exome sequencing in sporadic autism spectrum disorders identifies severe de novo mutations. Nat Genet. 43, 585-589 (2011).

8. O'Roak, B. J. et al. Sporadic autism exomes reveal a highly interconnected protein network of de novo mutations. Nature 485, 246-250 (2012).

9. Dingledine, R., Borges, K., Bowie, D. \& Traynelis, S. F. The glutamate receptor ion channels. Pharmacol Rev. 51, 57-61 (1999).

10. Toro, R. et al. Key role for gene dosage and synaptic homeostasis in autism spectrum disorders. Trends Genet. 26, 363-372 (2010).

11. Bourgeron, T. A synaptic trek to autism. Curr Opin Neurobiol. 19, 231-234 (2009).

12. Mayer, M. L. Glutamate receptors at atomic resolution. Nature. 440, 456-462 (2006).

13. Fetterolf, F. \& Foster, K. A. Regulation of Long-Term Plasticity Induction by the Channel and C-Terminal Domains of GluN2 Subunits. Mol Neurobiol. 44, 71-82 (2011).

14. Sprengel, R. et al. Importance of the intracellular domain of NR2 subunits for NMDA receptor function in vivo. Cell. 92, 279-289 (1998).

15. Maurano, M. T. et al. Systematic localization of common disease-associated variation in regulatory DNA. Science. 337, 1190-1195 (2012).

16. Xia, K. et al. Common genetic variants on 1 p13.2 associate with risk of autism. Mol Psychiatr. 19, 1212-1219 (2013).

17. Purcell, S. et al. PLINK: a tool set for whole-genome association and populationbased linkage analyses. Am J Hum Genet. 81, 559-575 (2007).

18. Barrett, J. C., Fry, B., Maller, J. \& Daly, M. J. Haploview: analysis and visualization of LD and haplotype maps. Bioinformatics. 21, 263-265 (2005).

\section{Acknowledgments}

We greatly thank all the patients and their family members who participated in this study. The research was supported by the National Basic Research Program of China (2012CB517900) and the National Natural Science Foundation of China (31301023, $81330027,81161120544,31400919$, and 81301172).

\section{Author contributions}

Study design: K.X. and H.G. Collecting the samples and performed the experiments: Y.P., J.C., J.O., Y.P., Q.L., Y.S., L.S., Y.L., Z.X., T.Z., S.L., Z.H. and J.Z. Data interpretation and analysis: Y.P., J.C. and H.G. Wrote the manuscript: H.G., Y.P. and J.C. All authors have read and approved the final manuscript.

\section{Additional information}

Competing financial interests: The authors declare no competing financial interests. How to cite this article: Pan, Y. et al. Association of genetic variants of GRIN2B with autism. Sci. Rep. 5, 8296; DOI:10.1038/srep08296 (2015).

This work is licensed under a Creative Commons Attribution 4.0 International License. The images or other third party material in this article are included in the article's Creative Commons license, unless indicated otherwise in the credit line; if the material is not included under the Creative Commons license, users will need to obtain permission from the license holder in order to reproduce the material. To view a copy of this license, visit http://creativecommons.org/licenses/by/4.0/ 